\title{
Accuracy and correlates of visual and verbal instruments assessing maternal perceptions of children's weight status: the Healthy Growth Study
}

\author{
George Moschonis ${ }^{1}$, Vasiliki latridi ${ }^{1}$, Christina Mavrogianni ${ }^{1}$, Paraskevi-Eirini Siatitsa ${ }^{1}$, \\ Aikaterini E Kyriakou ${ }^{1}$, Vasiliki Dede ${ }^{1}$, Georgia Skouli ${ }^{2}$, Afroditi Sakellaropoulou ${ }^{3}$ and \\ Yannis Manios ${ }^{1, *}$ \\ 'Department of Nutrition \& Dietetics, Harokopio University of Athens, 70 El. Venizelou Avenue, 17671 \\ Kallithea, Athens, Greece: ${ }^{2}$ 2nd Pediatric Department, Aristotle University of Thessaloniki, Thessaloniki, Greece: \\ ${ }^{3}$ Medical Center Zaglivery, Thessaloniki, Greece
}

Submitted 11 June 2010: Accepted 9 February 2011: First published online 24 May 2011

\begin{abstract}
Objective: To examine the accuracy of maternal ability to classify their children's weight status correctly using a verbal and a visual classification instrument and to detect significant correlates of maternal misperceptions.

Design: Cross-sectional study.

Setting: Primary schools in four counties from north, west, central and south Greece.

Subjects: A representative sample of 1858 primary-school children aged 9-13 years was examined. Two different instruments to assess maternal perceptions of their children's weight status, i.e. a verbal and a visual one, were used.

Results: Verbal and visual maternal underestimation rates of children's weight status were $15 \cdot 0 \%$ and $41 \cdot 3 \%$, respectively. The frequency of underestimation was much higher among overweight and obese children for both instruments. The highest underestimation rates of $87.9 \%$ and $82 \cdot 1 \%$ in overweight and obese boys, respectively, were obtained with the visual instrument. Multiple logistic regression modelling revealed that the likelihood of both verbal and visual maternal underestimation of their children's weight status was significantly higher for overweight mothers and for those with a lower educational level. Furthermore, children's male gender and a nanny or someone other than the mother as the child's primary caregiver were found to increase the odds of visual and verbal maternal underestimation of children's weight status, respectively.

Conclusions: The present study showed that the verbal instrument used to assess maternal perceptions of their children's weight status was more accurate compared with the visual one. However, both instruments showed that a considerable number of overweight and obese boys had their weight status underestimated by their mothers. Educating mothers to classify their children's weight status correctly might be a key factor for the implementation of successful childhood obesity prevention initiatives.
\end{abstract}

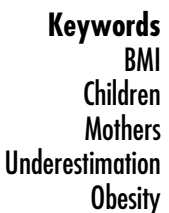

In many developed countries, the prevalence of childhood overweight has more than doubled over the last two decades ${ }^{(1)}$. Besides the manifestation of clinical signs of morbidity in overweight children, such as hypertension, hyperlipidaemia, etc. ${ }^{(2)}$, childhood overweight often tracks into adulthood ${ }^{(2,3)}$, when it becomes associated with an increased risk of mortality from chronic disease ${ }^{(4)}$. Changes in dietary and physical activity patterns are the primary causes for the current obesity epidemic and related chronic disease ${ }^{(5)}$. Specifically, the past few decades have brought marked lifestyle changes resulting in a decline in the time spent in physical activities, as well as in an increase in the consumption of energy-dense foods ${ }^{(5)}$. However, the aetiology of childhood overweight is probably more complex than the changes induced in lifestyle.

On the basis of recent research, many characteristics and lifestyle patterns that predispose children to overweight are established early in life and are greatly determined by family characteristics, including parents' dietary intake and physical activity patterns, parents' nutritional knowledge, siblings' interaction, etc. ${ }^{(6)}$. Furthermore, as 
parents are very influential in shaping early dietary and physical activity patterns in their children, their ability to recognize the problem of increased adiposity in their own child is another important determinant of childhood overweight $^{(7)}$. In general, it is critical for parents to be able to make a distinction between normal and abnormal levels of adiposity to ensure that appropriate preventive and corrective interventions are implemented.

However, several studies have shown that a significant number of parents are unable to correctly perceive their children's weight status when asked to make either a verbal or a visual estimation ${ }^{(8-21)}$, or both ${ }^{(22-24)}$. This observation implies that public health messages regarding the prevention of childhood obesity may not always reach parents, who often fail to identify the excessive body weight of their children. The factors that are associated with parental misperception of their child's weight status are currently under investigation. For example, some studies have shown that certain family characteristics such as ethnicity $^{(25,26)}$, child's gender ${ }^{(10,18,27)}$ and parental educational status ${ }^{(11,15)}$ may influence parental perception, whereas other studies did not find such associations ${ }^{(9,13)}$.

The objectives of the present study were: (i) to examine the accuracy of maternal ability to classify their children's weight status correctly using a verbal and a visual classification instrument; and (ii) to detect possible demographic, socio-economic, maternal and family correlates of maternal underestimation of their children's weight status.

\section{Subjects and methods}

\section{Sampling}

The 'Healthy Growth Study' was a large-scale crosssectional epidemiological study initiated in May 2007 and completed in June 2009. Approval to conduct the study was granted by the Greek Ministry of National Education and by the Ethical Committee of Harokopio University of Athens. The study population comprised schoolchildren aged 9-13 years attending the fifth and sixth grades in primary schools located in municipalities within the wider regions of the counties of Attica, Aitoloakarnania, Thessaloniki and Iraklio. The sampling of schools was random, multistage and stratified by parental educational level and by the total population of students attending schools within these municipalities. More specifically, the municipalities in the counties under study were divided into three groups on the basis of the average educational level of their adult population (25-65 years of age), which was estimated from data provided by the National Statistical Service of Greece (Census 2001). This procedure yielded two parental educational cut-off points that allowed us to categorize municipalities into three different socio-economic levels (SEL), i.e. higher, medium and lower SEL. Consequently, on the basis of data from the National Statistical Service of Greece, a certain number of municipalities, proportional to the size of their pre-adolescent population (9-14 years of age), were randomly selected from each one of these three SEL groups.

An appropriate number of schools were randomly selected from each one of these municipalities in relation to the population of schoolchildren registered in the fifth and sixth grades in each municipality, on the basis of data obtained from the Greek Child Institute. All seventy-seven primary schools that were invited to participate in the present study responded positively. More specifically, an extended letter explaining the aims of the present study and a consent form for taking full measurements were provided to all parents or guardians having a child in these schools. Parents who agreed to the participation of their children in the study had to sign the consent form and provide their contact details. Signed parental consent forms were collected for 2655 out of 4145 children (response rate: $64 \cdot 1 \%$ ).

\section{Antbropometry and physical examination}

Participants from each primary school underwent a physical examination by two trained members of the research team. Anthropometric measurements were obtained from all regions using the same protocol and equipment that was calibrated with standardized weights and rods, according to the calibration procedures described by the manufacturers. The physical examination included basic anthropometric measurements, i.e. weight and height. Weight was measured to the nearest $10 \mathrm{~g}$ using a Seca digital scale (Seca Alpha, Model 770; Hamburg, Germany). Participants were weighed without shoes in the minimum clothing possible. Height was measured to the nearest $0 \cdot 1 \mathrm{~cm}$ using a commercial stadiometer (Leicester Height Measure; Invicta Plastics Ltd, Oadby, UK) with participants not wearing shoes, their shoulders in a relaxed position, their arms hanging freely and their head aligned in Frankfort plane. Weight and height were converted to BMI using Quetelet's equation (weight $(\mathrm{kg}) /$ height $^{2}\left(\mathrm{~m}^{2}\right)$ ). The international age- and sex-specific cut-off points proposed by Cole et al. ${ }^{(28,29)}$ for children and adolescents aged 2-18 years were used to categorize participants as 'underweight', 'normal weight', 'overweight' or 'obese'. Regarding physical examination, one well-trained and experienced female paediatrician in each prefecture determined pubertal maturation (Tanner stage) after a thorough visual inspection of breast development in girls and genital development in boys ${ }^{(30)}$.

\section{Demographic, socio-economic data, maternal and family characteristics}

Data on the socio-economic and demographic backgrounds of families having at least one child participating in the study were collected from the primary caregiver (most preferably from the mother) during scheduled faceto-face interviews at school. With regard to those primary 
caregivers who were unable to attend the meetings (approximately 5\% of the total sample), data were collected via telephonic interviews. All interviews were conducted by a research team of ten members who were rigorously trained to minimize the interviewer's effect by using a standardized questionnaire. More specifically, the data collected by primary caregivers included: demographic characteristics such as parent's age and nationality; socio-economic characteristics such as educational level (years of education) and mean annual family income over the last 3 years ( $€ /$ year); lifestyle characteristics such as smoking habits; and anthropometric characteristics such as mother's height and weight. Mother's age was then classified into three different tertiles. Mother's weight and height were converted to BMI using Quetelet's equation. The International Obesity Task Force cut-off points for adults were used for the categorization of mother's weight status as: 'underweight' $\left(\mathrm{BMI}<18.5 \mathrm{~kg} / \mathrm{m}^{2}\right)$, 'normal weight' $\left(18.5 \leq \mathrm{BMI}<25 \cdot 0 \mathrm{~kg} / \mathrm{m}^{2)}\right.$, 'overweight' $\left(25 \cdot 0 \leq \mathrm{BMI}<30 \cdot 0 \mathrm{~kg} / \mathrm{m}^{2}\right)$ and 'obese' $\left(\mathrm{BMI} \geq 30 \cdot 0 \mathrm{~kg} / \mathrm{m}^{2}\right)$. Finally, children's age was calculated on the basis of their date of birth as recorded in the children's paediatric medical records.

\section{Primary caregivers' perceptions of their children's weight status}

Maternal perceptions of their children's weight status and body image were assessed using a verbal and a visual classification instrument, respectively. Regarding verbal classification of a child's weight status, the child's primary caregivers were asked to provide their answers to the following two questions: 'According to your perception, what is the current weight of your child?'; and 'According to your perception, what is the current height of your child?' Children's weight and height, as perceived by parents, were also converted to BMI by using Quetelet's equation and thereafter their weight status was classified into different weight categories (underweight, normal weight, overweight and obese) using Cole's cut-off points ${ }^{(28,29)}$. Regarding visual classification of the children's body shape, this was estimated using the 'Children's Body Image Scale' as proposed by Truby and Paxton ${ }^{(31)}$. In brief, two sets of age- and gender-specific photographs were available. Each photograph corresponded to a 10-year-old boy or girl with a BMI on or around each of the seven conventional 1979 National Center for Health Statistics (NCHS) percentiles (i.e. 3rd, 10th, 25th, 50th, 75th, 90th and 97 th $)^{(32)}$. More specifically, in each set, one photograph corresponded to an underweight child (3rd percentile), four photographs to children within the normal BMI-forage percentile range (10th-75th percentiles), one photograph to an overweight child (90th percentile) and the last photograph to an obese child (97th percentile). After presenting this set of photographs in random order, mothers were asked to choose the photograph that most resembled their child's body shape.

\section{Statistical analysis}

All variables presented were categorical and expressed as percentages. Associations between categorical variables were examined using the $\chi^{2}$ or Fisher's exact test whenever appropriate. Cross-classification analysis was further carried out to identify the proportion of participants classified correctly or incorrectly by the verbal and visual classification methods. The two-sample Z-test was also used to compare percentages of correct or incorrect verbal and visual classifications of children's actual weight status. Univariate logistic regression analyses were conducted to test the crude bivariate associations of several demographic, socio-economic and family status variables per se with mothers' verbal and visual underestimation of their children's weight status. Furthermore, multiple logistic regression analysis was conducted to identify which of the demographic, socio-economic, maternal and family status variables were independently associated with maternal underestimation of children's weight status. From all these analyses OR and 95\% CI were computed. All reported $P$ values were based on two-sided tests. The level of statistical significance was set at $P<0 \cdot 05$. The Statistical Package for the Social Sciences statistical software package version $13 \cdot 0$ (SPSS Inc., Chicago, IL, USA) was used to conduct all statistical analyses.

\section{Results}

Complete socio-economic, demographic, maternal, family status, verbal and visual classification data were collected for 1858 children $(51 \cdot 1 \%$ girls and $48.9 \%$ boys) from the 2665 children whose parents had signed the consent forms. This was mainly because of the addition of the visual assessment tool in the parental questionnaire during the course of the study when approximately 800 children and their parents had already been measured. Nevertheless, the study sample of 1858 children examined in the present study was adequate to provide enough statistical power. Approximately $45 \%$ of the participating children were normal weight, $31 \%$ were overweight and $11 \%$ were obese. Regarding the characteristics of mothers, 39.5\% had 9-12 years of education whereas $40.4 \%$ had $>12$ years. The majority of participating mothers were of Greek nationality (83.6\%) and were married (89.5\%). Significant associations were observed between children's weight status and certain maternal characteristics. More specifically, childhood obesity was found to be positively associated with maternal obesity and smoking status, with a greater proportion of mothers who were obese or smokers having an obese child ( $P<0 \cdot 001$, respectively). On the other hand, maternal educational levels were negatively associated with childhood obesity, since the percentage of obese children was significantly lower for mothers with a higher level of education than for mothers with a medium 
Table 1 Demographic, socio-economic, maternal and family descriptive characteristics of the study population presented by weight groups

\begin{tabular}{|c|c|c|c|c|c|c|}
\hline & \multirow[b]{2}{*}{$\begin{array}{c}\text { Total } \\
(n \text { 1858) }\end{array}$} & \multicolumn{4}{|c|}{ Children's actual weight status } & \multirow[b]{3}{*}{$P+$} \\
\hline & & $\begin{array}{l}\text { Underweight } \\
\text { (n 56) }\end{array}$ & $\begin{array}{l}\text { Normal weight } \\
\quad(n \text { 1019) }\end{array}$ & $\begin{array}{l}\text { Overweight } \\
\text { ( } n 574)\end{array}$ & $\begin{array}{l}\text { Obese } \\
(n \text { 209) }\end{array}$ & \\
\hline & $\%$ & $\%$ & $\%$ & $\%$ & $\%$ & \\
\hline \multicolumn{7}{|l|}{ Child's age (years) } \\
\hline $9-11$ & $41 \cdot 8$ & $2 \cdot 6$ & $54 \cdot 8$ & $30 \cdot 9$ & $11 \cdot 7$ & \multirow[t]{2}{*}{$0 \cdot 005$} \\
\hline $11-13$ & $58 \cdot 2$ & $3 \cdot 3$ & $54 \cdot 9$ & $30 \cdot 9$ & $10 \cdot 9$ & \\
\hline \multicolumn{7}{|l|}{ Child's gender } \\
\hline Female & $51 \cdot 1$ & $3 \cdot 6$ & $57 \cdot 2$ & $30 \cdot 1$ & $9 \cdot 1$ & \multirow[t]{2}{*}{0.005} \\
\hline Male & $48 \cdot 9$ & $2 \cdot 4$ & $52 \cdot 4$ & $31 \cdot 7$ & $13 \cdot 5$ & \\
\hline \multicolumn{7}{|l|}{ Child's primary caregiver } \\
\hline Mother & $53 \cdot 3$ & $3 \cdot 2$ & $56 \cdot 0$ & $29 \cdot 6$ & $11 \cdot 2$ & \multirow[t]{3}{*}{0.298} \\
\hline Grandparents & $40 \cdot 7$ & $2 \cdot 6$ & $53 \cdot 7$ & $31 \cdot 4$ & $12 \cdot 3$ & \\
\hline Nanny or someone else & $6 \cdot 0$ & $4 \cdot 6$ & $51 \cdot 4$ & $37 \cdot 6$ & $6 \cdot 4$ & \\
\hline \multicolumn{7}{|l|}{ Mother's age category } \\
\hline Lower (<38 years old) & $39 \cdot 8$ & $2 \cdot 9$ & $54 \cdot 4$ & $30 \cdot 2$ & $12 \cdot 5$ & \multirow[t]{3}{*}{0.541} \\
\hline Medium (38-42 years old) & 34.5 & $2 \cdot 3$ & $55 \cdot 1$ & $31 \cdot 6$ & $11 \cdot 0$ & \\
\hline Higher (>42 years old) & $26 \cdot 6$ & $4 \cdot 1$ & $55 \cdot 4$ & $30 \cdot 8$ & $9 \cdot 7$ & \\
\hline \multicolumn{7}{|l|}{ Mother's BMI $\left(\mathrm{kg} / \mathrm{m}^{2}\right)$} \\
\hline Underweight & $2 \cdot 6$ & $14 \cdot 3$ & $63 \cdot 3$ & $20 \cdot 4$ & $2 \cdot 0$ & \multirow[t]{4}{*}{$<0.001$} \\
\hline Normal weight & $57 \cdot 9$ & $3 \cdot 4$ & $60 \cdot 2$ & $29 \cdot 2$ & $7 \cdot 3$ & \\
\hline Overweight & $26 \cdot 9$ & $2 \cdot 2$ & $50 \cdot 5$ & $31 \cdot 5$ & $15 \cdot 8$ & \\
\hline Obese & $12 \cdot 6$ & $0 \cdot 9$ & $38 \cdot 2$ & $39 \cdot 5$ & $21 \cdot 5$ & \\
\hline \multicolumn{7}{|c|}{ Mother's educational level (years of education) } \\
\hline$<9$ & $20 \cdot 2$ & $3 \cdot 7$ & $49 \cdot 6$ & $31 \cdot 2$ & $15 \cdot 5$ & \multirow[t]{3}{*}{0.007} \\
\hline $9-12$ & $39 \cdot 5$ & $3 \cdot 1$ & $54 \cdot 4$ & $30 \cdot 2$ & $12 \cdot 3$ & \\
\hline$>12$ & $40 \cdot 4$ & $2 \cdot 5$ & $57 \cdot 9$ & $31 \cdot 5$ & $8 \cdot 1$ & \\
\hline \multicolumn{7}{|l|}{ Mother's nationality } \\
\hline Greek & $83 \cdot 6$ & $3 \cdot 1$ & $54 \cdot 1$ & $31 \cdot 5$ & $11 \cdot 3$ & \multirow[t]{2}{*}{0.462} \\
\hline Non-Greek & $16 \cdot 4$ & $2 \cdot 6$ & $58 \cdot 9$ & $27 \cdot 6$ & $10 \cdot 9$ & \\
\hline \multicolumn{7}{|l|}{ Mother's smoking habits } \\
\hline Smoker & $38 \cdot 2$ & $2 \cdot 5$ & $50 \cdot 4$ & $32 \cdot 1$ & $14 \cdot 9$ & \multirow[t]{2}{*}{$<0 \cdot 001$} \\
\hline Non-smoker & $61 \cdot 8$ & $3 \cdot 3$ & $57 \cdot 6$ & $30 \cdot 1$ & $9 \cdot 0$ & \\
\hline \multicolumn{7}{|l|}{ Mother's marital status } \\
\hline Married & $89 \cdot 5$ & $3 \cdot 1$ & $55 \cdot 0$ & $30 \cdot 7$ & $11 \cdot 2$ & \multirow[t]{4}{*}{0.943} \\
\hline Not married & $1 \cdot 2$ & $4 \cdot 3$ & $52 \cdot 2$ & $34 \cdot 8$ & $8 \cdot 7$ & \\
\hline Divorced & $7 \cdot 5$ & $1 \cdot 4$ & $56 \cdot 4$ & $30 \cdot 7$ & $11 \cdot 4$ & \\
\hline Widowed & $1 \cdot 7$ & $3 \cdot 1$ & $43 \cdot 8$ & $37 \cdot 5$ & $15 \cdot 6$ & \\
\hline \multicolumn{7}{|l|}{ SEL of residential area } \\
\hline Lower & $24 \cdot 8$ & $3 \cdot 3$ & $57 \cdot 2$ & $27 \cdot 6$ & $12 \cdot 0$ & \multirow[t]{3}{*}{$0 \cdot 187$} \\
\hline Medium & $33 \cdot 7$ & $3 \cdot 7$ & $51 \cdot 9$ & $31 \cdot 8$ & $12 \cdot 6$ & \\
\hline Higher & $41 \cdot 6$ & $2 \cdot 3$ & $55 \cdot 8$ & $32 \cdot 1$ & $9 \cdot 7$ & \\
\hline Family income (€/year) & & & & & & \\
\hline$<12000$ & $22 \cdot 0$ & $2 \cdot 9$ & $56 \cdot 7$ & $27 \cdot 9$ & $12 \cdot 5$ & $0 \cdot 766$ \\
\hline $12000-30000$ & $50 \cdot 6$ & $3 \cdot 1$ & $53 \cdot 7$ & $31 \cdot 8$ & $11 \cdot 4$ & \\
\hline$>30000$ & $27 \cdot 4$ & $2 \cdot 9$ & $55 \cdot 4$ & $31 \cdot 6$ & $10 \cdot 0$ & \\
\hline
\end{tabular}

SEL, socio-economic level.

tAssociations between weight categories and all other categorical variables included in the table were tested using Pearson's $\chi^{2}$ or Fisher's exact test whenever appropriate.

or lower educational level $(P=0 \cdot 007)$. Further descriptive characteristics of the children and their mothers are summarized in Table 1.

Table 2 illustrates the percentages of maternal verbal and visual classification of their children's weight status by percentages of their children's actual weight status. More specifically, correct classification of children's actual weight status was significantly higher for overweight girls $(67 \cdot 7 \% v \cdot 22 \cdot 7 \%, P<0 \cdot 001)$ and boys $(72 \cdot 4 \% v \cdot 9 \cdot 7 \%$, $P<0.001)$, as well as for obese boys $(60.9 \% v \cdot 17 \cdot 9 \%$, $P<0 \cdot 001$ ), when mothers estimated their children's weight status using the verbal rather than the visual classification instrument. Furthermore, the percentages of obese girls $(4 \cdot 1 \% v \cdot 20 \cdot 9 \%, P<0 \cdot 01)$ and boys $(1 \cdot 8 \% v$. $48 \%, P<0 \cdot 001)$ who were misclassified by their mothers as normal weight were significantly lower when mothers tried to perceive their children's weight status using the verbal rather than the visual classification instrument. Overall, a significant percentage of mothers tended to misclassify their overweight or obese child, especially when classifying them visually.

Table 3 summarizes the crude OR and 95\% CI for maternal verbal underestimation of their children's weight status. More specifically, mothers with $9-12$ and $<9$ years of education were $1.47(95 \%$ CI $1 \cdot 07,2 \cdot 00)$ and $1.72(95 \%$ CI $1 \cdot 20,2 \cdot 47)$ times more likely to verbally underestimate 
Table 2 Maternal verbal and visual classification of children's weight status by children's actual weight status

\begin{tabular}{|c|c|c|c|c|c|c|c|c|}
\hline \multirow{4}{*}{$\begin{array}{l}\text { Primary caregivers' classification } \\
\text { of their children's weight status }\end{array}$} & \multicolumn{8}{|c|}{ Children's actual weight status } \\
\hline & \multicolumn{2}{|c|}{ Underweight $(n 56)$} & \multicolumn{2}{|c|}{ Normal weight ( $n$ 1019) } & \multicolumn{2}{|c|}{ Overweight ( $n$ 574) } & \multicolumn{2}{|c|}{ Obese $(n$ 209) } \\
\hline & Girls & Boys & Girls & Boys & Girls & Boys & Girls & Boys \\
\hline & $\%$ & $\%$ & $\%$ & $\%$ & $\%$ & $\%$ & $\%$ & $\%$ \\
\hline \multicolumn{9}{|l|}{ Verbal } \\
\hline Underweight & $61 \cdot 3$ & $42 \cdot 9$ & $4 \cdot 1^{\star \star \star}$ & $4 \cdot 8^{*}$ & 0.0 & $0 \cdot 8$ & 0.0 & 0.0 \\
\hline Normal weight & $35 \cdot 5$ & $57 \cdot 1$ & 89.9 & $84 \cdot 8^{*}$ & $29 \cdot 1^{\star \star \star}$ & $21 \cdot 2^{\star}$ & $4 \cdot 1^{\star *}$ & $1 \cdot 8^{*}$ \\
\hline Overweight & $3 \cdot 2$ & 0.0 & $5 \cdot 8^{\star \star \star}$ & $9 \cdot 7^{\star}$ & $67 \cdot 7^{\star \star \star}$ & $72 \cdot 4^{\star}$ & $41 \cdot 9$ & $37 \cdot 3$ \\
\hline Obese & $0 \cdot 0$ & 0.0 & $0 \cdot 2$ & 0.7 & $3 \cdot 1$ & $5 \cdot 1$ & $54 \cdot 1$ & $60 \cdot 9^{\star}$ \\
\hline \multicolumn{9}{|l|}{ Visual } \\
\hline Underweight & $47 \cdot 1$ & $50 \cdot 0$ & $11 \cdot 2^{\star \star \star}$ & $25 \cdot 4^{*}$ & 0.3 & $0 \cdot 7$ & $1 \cdot 2$ & 0.0 \\
\hline Normal weight & $52 \cdot 9$ & $50 \cdot 0$ & $87 \cdot 3$ & $72 \cdot 9^{\star}$ & $67 \cdot 1^{\star \star \star}$ & $87 \cdot 2^{\star}$ & $20 \cdot 9^{\star \star}$ & $48 \cdot 0^{\star}$ \\
\hline Overweight & 0.0 & $0 \cdot 0$ & $1 \cdot 1^{\star \star \star}$ & $1 \cdot 3^{*}$ & $22 \cdot 7^{\star \star \star}$ & $9 \cdot 7^{\star}$ & $30 \cdot 2$ & $34 \cdot 1$ \\
\hline Obese & $0 \cdot 0$ & 0.0 & $0 \cdot 4$ & 0.4 & $9 \cdot 8$ & $2 \cdot 4$ & $47 \cdot 7$ & $17 \cdot 9^{\star}$ \\
\hline
\end{tabular}

Percentages in bold indicate correct weight status classification.

${ }^{\star} P<0.001$ for differences between verbal and visual classification of girls' weight status, based on the two-sample Z-test.

${ }^{\star \star} P<0.01$ and ${ }^{* \star \star} P<0.001$ for differences between verbal and visual classification of boys' weight status, based on the two-sample $Z$-test.

Table 3 Logistic regression analyses for the association between maternal verbal underestimation of children's weight status with demographic, socio-economic, maternal and family characteristics of the study population

\begin{tabular}{|c|c|c|c|c|}
\hline \multirow[b]{2}{*}{ Independent variable } & \multicolumn{4}{|c|}{ Verbal underestimation of the child's weight category } \\
\hline & Crude OR & $95 \% \mathrm{Cl}$ & Adjusted OR & $95 \% \mathrm{Cl}$ \\
\hline \multicolumn{5}{|c|}{ Mother's educational level (years of education) } \\
\hline$>12$ & Ref. & & Ref. & \\
\hline $9-12$ & $1 \cdot 47$ & $1 \cdot 07,2 \cdot 00$ & $1 \cdot 52$ & $1 \cdot 10,2 \cdot 11$ \\
\hline$<9$ & $1 \cdot 72$ & $1 \cdot 20,2 \cdot 47$ & $1 \cdot 89$ & $1 \cdot 30,2 \cdot 77$ \\
\hline \multicolumn{5}{|l|}{ Mother's weight status } \\
\hline Under/normal weight & Ref. & & Ref. & \\
\hline Overweight & $1 \cdot 44$ & $1.06,1.95$ & $1 \cdot 48$ & $1 \cdot 08,2 \cdot 02$ \\
\hline Obese & $1 \cdot 30$ & $0.85,1.95$ & $1 \cdot 26$ & $0.83,1.93$ \\
\hline \multicolumn{5}{|l|}{ Child's primary caregiver } \\
\hline Mother & Ref. & & Ref. & \\
\hline Grandparents & 0.97 & $0.73,1 \cdot 29$ & $1 \cdot 05$ & $0 \cdot 78,1 \cdot 41$ \\
\hline Nanny or someone else & $1 \cdot 62$ & $1 \cdot 02,2 \cdot 72$ & $1 \cdot 70$ & $1 \cdot 00,2 \cdot 89$ \\
\hline \multicolumn{5}{|l|}{ SEL of residential area } \\
\hline Lower & Ref. & & Ref. & \\
\hline Medium & $1 \cdot 38$ & $1.03,1.97$ & $1 \cdot 41$ & $0.98,2.03$ \\
\hline Higher & $1 \cdot 08$ & $0.75,1.54$ & $1 \cdot 18$ & $0 \cdot 81,1 \cdot 70$ \\
\hline \multicolumn{5}{|l|}{ Mother's age category } \\
\hline Lower $(<38$ years old $)$ & Ref. & & Ref. & \\
\hline Medium (38-42 years old) & $0 \cdot 82$ & $0 \cdot 60,1 \cdot 12$ & $0 \cdot 87$ & $0 \cdot 62,1 \cdot 21$ \\
\hline Higher ( $>42$ years old) & 0.73 & $0.52,0.98$ & 0.77 & $0 \cdot 54,1 \cdot 11$ \\
\hline \multicolumn{5}{|l|}{ Child's gender } \\
\hline Male & Ref. & & & \\
\hline Female & $1 \cdot 04$ & $0 \cdot 8,1 \cdot 37$ & & \\
\hline \multicolumn{5}{|l|}{ Mother's smoking habit } \\
\hline Non-smoker & Ref. & & & \\
\hline Smoker & $1 \cdot 10$ & $0.84,1.46$ & & \\
\hline \multicolumn{5}{|l|}{ Maternal marital status } \\
\hline Married & Ref. & & & \\
\hline Not married & 0.33 & $0 \cdot 44,2 \cdot 50$ & & \\
\hline Divorced & $1 \cdot 10$ & $0 \cdot 66,1 \cdot 80$ & & \\
\hline Widowed & 0.50 & $0 \cdot 11,2 \cdot 10$ & & \\
\hline \multicolumn{5}{|l|}{ Family incomet ( $€ /$ year) } \\
\hline$<12000$ & Ref. & & & \\
\hline $12000-30000$ & 0.94 & $0 \cdot 67,1 \cdot 33$ & & \\
\hline$>30000$ & $0 \cdot 85$ & $0.57,1.25$ & & \\
\hline
\end{tabular}

SEL, socio-economic level; Ref., reference category.

Values in bold indicate statistically significant OR.

${ }^{*}$ Adjusted for the number of people in the household.

their children's weight category compared with mothers with $>12$ years of education. Furthermore, overweight mothers were $1 \cdot 44(95 \%$ CI $1 \cdot 06,1 \cdot 95)$ times more likely to verbally underestimate their children's weight status compared with under/normal-weight mothers. The likelihood of maternal verbal underestimation of their children's 
Table 4 Logistic regression analyses for the association of maternal visual underestimation of children's weight status with demographic, socio-economic, maternal and family characteristics of the study population

\begin{tabular}{|c|c|c|c|c|}
\hline \multirow[b]{2}{*}{ Independent variable } & \multicolumn{4}{|c|}{ Visual underestimation of the child's weight category } \\
\hline & Crude OR & $95 \% \mathrm{Cl}$ & Adjusted OR & $95 \% \mathrm{Cl}$ \\
\hline \multicolumn{5}{|c|}{ Mother's educational level (years of education) } \\
\hline$>12$ & Ref. & & Ref. & \\
\hline $9-12$ & $0 \cdot 91$ & $0 \cdot 74,1 \cdot 12$ & $0 \cdot 90$ & $0 \cdot 72,1 \cdot 13$ \\
\hline$<9$ & 0.72 & $0.55,0.93$ & 0.80 & $0.60,0.99$ \\
\hline \multicolumn{5}{|l|}{ Mother's weight status } \\
\hline Under/normal weight & Ref. & & Ref. & \\
\hline Overweight & $1 \cdot 38$ & $1 \cdot 11,1 \cdot 71$ & 1.48 & $1 \cdot 18,1 \cdot 85$ \\
\hline Obese & $1 \cdot 82$ & $1 \cdot 37,2 \cdot 42$ & 1.95 & $1 \cdot 45,2 \cdot 62$ \\
\hline \multicolumn{5}{|l|}{ Child's gender } \\
\hline Male & Ref. & & Ref. & \\
\hline Female & 0.42 & $0.34,0.50$ & 0.40 & $0.33,0.49$ \\
\hline \multicolumn{5}{|l|}{ SEL of residential area } \\
\hline Lower & Ref. & & Ref. & \\
\hline Medium & $1 \cdot 32$ & $1 \cdot 03,1 \cdot 70$ & $1 \cdot 28$ & $0.99,1.66$ \\
\hline Higher & $1 \cdot 25$ & $0.98,1.58$ & $1 \cdot 15$ & $0.90,1.48$ \\
\hline \multicolumn{5}{|l|}{ Family incomet (€/year) } \\
\hline$<12000$ & Ref. & & Ref. & \\
\hline $12000-30000$ & $1 \cdot 14$ & $0 \cdot 90,1 \cdot 45$ & $1 \cdot 01$ & $0 \cdot 78,1 \cdot 31$ \\
\hline$>30000$ & $1 \cdot 30$ & $1 \cdot 00,1 \cdot 70$ & 1.09 & $0.80,1 \cdot 49$ \\
\hline \multicolumn{5}{|l|}{ Mother's nationality } \\
\hline Greek & Ref. & & Ref. & \\
\hline Non-Greek & 0.78 & $0.61,0.96$ & $0 \cdot 86$ & $0 \cdot 65,1 \cdot 14$ \\
\hline \multicolumn{5}{|l|}{ Child's primary caregiver } \\
\hline Mother & Ref. & & & \\
\hline Grandparents & $1 \cdot 08$ & $0 \cdot 89,1 \cdot 31$ & & \\
\hline Nanny or someone else & $1 \cdot 29$ & $0.86,1.92$ & & \\
\hline \multicolumn{5}{|l|}{ Mother's age category } \\
\hline Lower $(<38$ years old $)$ & Ref. & & & \\
\hline Medium (38-42 years old) & 1.07 & $0 \cdot 86,1 \cdot 33$ & & \\
\hline Higher ( $>42$ years old) & $1 \cdot 21$ & $0.96,1.52$ & & \\
\hline \multicolumn{5}{|l|}{ Mother's smoking habit } \\
\hline Non-smoker & Ref. & & & \\
\hline Smoker & 1.09 & $0 \cdot 90,1 \cdot 31$ & & \\
\hline \multicolumn{5}{|l|}{ Maternal marital status } \\
\hline Married & Ref. & & & \\
\hline Not married & $1 \cdot 09$ & $0 \cdot 47,2 \cdot 50$ & & \\
\hline Divorced & $1 \cdot 22$ & $0 \cdot 86,1 \cdot 73$ & & \\
\hline Widowed & $0 \cdot 74$ & $0 \cdot 35,1 \cdot 55$ & & \\
\hline Tanner stage & $1 \cdot 04$ & $0 \cdot 94,1 \cdot 15$ & & \\
\hline
\end{tabular}

SEL, socio-economic level; Ref., reference category.

Values in bold indicate statistically significant OR.

tAdjusted for the number of people in the household.

weight status was also higher when the children's primary caregiver was a nanny or someone other than the mother $(\mathrm{OR}=1 \cdot 62 ; 95 \% \mathrm{CI} 1 \cdot 02,2 \cdot 72)$ and lower for older $(>42$ years old) than for younger ( $<38$ years old) mothers $(\mathrm{OR}=0.73 ; 95 \%$ CI $0.53,0.98)$. Table 3 also presents the adjusted OR and 95\% CI derived from the multivariate logistic regression analysis, including in the same model all variables that were found to be significantly related to maternal verbal underestimation of their children's weight category at a univariate level. On the basis of the results derived from this analysis, lower (OR $=1 \cdot 89 ; 95 \%$ CI $1 \cdot 30$, $2 \cdot 77)$ and medium maternal educational levels ( $\mathrm{OR}=1 \cdot 52$; $95 \%$ CI $1 \cdot 10,2 \cdot 11)$, maternal overweight (OR $=1 \cdot 48$; $95 \%$ CI $1 \cdot 08,2 \cdot 02)$ and nanny or someone else as the child's primary caregiver $(\mathrm{OR}=1 \cdot 70 ; 95 \% \mathrm{CI} 1 \cdot 00,2 \cdot 89)$ remained significantly associated with maternal verbal underestimation of their children's weight status.
Table 4 summarizes the crude and adjusted OR and $95 \%$ CI for maternal visual underestimation of their children's weight status. More specifically, mothers with $<9$ years of education, mothers with female children and those of non-Greek nationality were 0.72 (95\% CI 0.55 , $0.93), 0.42(95 \%$ CI $0.35,0.50)$ and 0.78 (95\% CI 0.61, 0.96) times less likely to visually underestimate their children's weight status compared with mothers with $>12$ years of education, mothers with male children and those of Greek nationality, respectively. On the other hand, overweight $(\mathrm{OR}=1 \cdot 38 ; 95 \% \mathrm{CI} 1 \cdot 11,1 \cdot 71)$ and obese $(\mathrm{OR}=1 \cdot 82 ; 95 \% \mathrm{CI} 1 \cdot 37,2 \cdot 42)$ mothers were more likely to visually underestimate their children's weight status in comparison with under/normal-weight mothers. Moreover, mothers living in areas of medium SEL (OR $=$ 1.32; $95 \%$ CI $1.03,1.70)$ and mothers with an annual family income of $>€ 30000(\mathrm{OR}=1 \cdot 30 ; 95 \%$ CI $1 \cdot 00$, 
1.70) were also more likely to visually underestimate their children's weight status compared with mothers living in areas of lower SEL and mothers with an annual family income of $<€ 12000$, respectively. Table 4 also presents the adjusted OR and $95 \% \mathrm{CI}$ derived from the multivariate logistic regression analysis, including in the same model all variables that were found to be significantly related to maternal verbal underestimation of their children's weight category at a univariate level. On the basis of the results derived from the present analysis, the factors that remained statistically significantly associated with maternal visual underestimation of their children's weight status were maternal lower educational level $(\mathrm{OR}=0 \cdot 80$; $95 \%$ CI 0.60, 0.99), maternal overweight (OR $=1 \cdot 48 ; 95 \%$ CI $1 \cdot 18,1 \cdot 85)$ and obesity $(\mathrm{OR}=1.95 ; 95 \%$ CI 1.45 , $2 \cdot 62)$ and female gender of children $(\mathrm{OR}=0 \cdot 40 ; 95 \% \mathrm{CI}$ $0 \cdot 33,0 \cdot 49)$.

\section{Discussion}

The present study used two different instruments to assess maternal perceptions of their children's weight status, i.e. a verbal and a visual one. The verbal instrument proved to be more accurate than the visual one in classifying children's actual weight status correctly. More specifically, $15 \cdot 0 \%$ of mothers verbally underestimated their children's weight status, mainly by under-reporting their children's actual weight and much less by overreporting their children's height. On the other hand, $41 \cdot 3 \%$ of mothers visually underestimated their children's weight status when they were asked to choose one photograph representing body images of boys and girls in different BMI-for-age percentiles that most resembled their children's body shape (the aforementioned percentages are not shown in the tables). These underestimation rates were found to be even higher for overweight and obese children compared with normal-weight children, reaching $87 \cdot 9 \%$ and $82 \cdot 1 \%$, respectively, in overweight and obese boys, whose weight status was visually assessed by their mothers. These findings were in agreement with those reported by other studies that examined parental perceptions of their children's weight status $^{(8-13,15,16,20-22)}$. Nevertheless, the verbal instruments used in these studies to assess parental perceptions were not entirely comparable to those used in the present study. Most of the other studies used a verbal classification scale to assess parental perceptions of their children's weight status, mostly with the following question or with one very similar to it: 'How would you describe your child's weight at the moment?', and with one of the answers: underweight, normal weight or about the right weight, overweight and obese ${ }^{(8-13,15,16)}$. Furthermore, sex-specific sketches or silhouettes corresponding to children of different weight- or BMI-for-age percentiles were used as visual classification scales by two other studies ${ }^{(20,21)}$, whereas both verbal and visual instruments were used in only one previous study ${ }^{(22)}$.

In only one other study were caregivers asked to report their children's actual weight and height ${ }^{(33)}$. In that study, the accuracy of the verbal classification of children's weight status was comparable to that reported by the present study with respect to underweight, normalweight and obese children, whereas a lower accuracy was observed for overweight children. Two other studies that used verbal and visual classification instruments, similar to those used in the present study, have failed to report higher accuracy of the verbal classification instrument, whereas others have provided some mixed findings. More specifically, according to the study by Eckstein et $a l^{(24)}$, the visual classification instrument (sketches) assessed children's weight status more accurately compared with the verbal classification instrument (word description). Furthermore, the study by Chaimovitz et $a l^{(23)}$, which was designed to assess the accuracy of parents' and paediatricians' perceptions of children's weight status, showed that parents displayed higher accuracy using the verbal classification instrument (word description), whereas doctors displayed higher accuracy with the visual classification instrument (sketches).

According to Maynard et al. ${ }^{(16)}$, the observation from several studies that mothers tend to underestimate their children's excessive body weight may reflect their reluctance to admit that their children are overweight or their lack of understanding of what 'overweight' means. Thus, recording and understanding maternal perceptions about their children's weight status, as well as the determinants of these perceptions, would be a key step in forming effective links between health professionals and parents $^{(29)}$ that could help overweight prevention programmes be more effective by focusing on the main concern of parents, which is maintenance of 'good health' in their children rather than 'good weight'(34).

In the present study several factors were found to be independently (i.e. at a multivariate level) associated with the likelihood of maternal verbal or visual underestimation of their children's weight status (Tables 3 and 4). Maternal educational level was found to be significantly associated with both verbal and visual maternal underestimation of children's weight status, although in a reverse direction. More specifically, the findings of the present study revealed that mothers with a lower educational level were more likely to make a verbal underestimation but less likely to make a visual underestimation of their children's weight status. The negative association observed between verbal underestimation and maternal educational level is in line with the findings from other similar studies ${ }^{(8,15)}$, whereas a possible interpretation for this association could be the fact that higher educational level may imply a better knowledge of normal body weight and height in children ${ }^{(15)}$. In addition, more educated mothers are probably more likely to be aware of the physical, social and 
emotional consequences of obesity. Nevertheless, no other study has reported an increased likelihood of maternal visual underestimation of their children's weight status, making an interpretation of this finding rather difficult.

Furthermore, overweight and obese mothers were significantly more likely to both verbally and visually underestimate their children's weight status. These findings were in agreement with those reported by other investigators who examined parental perceptions of their children's weight status $^{(14,25,26)}$, probably also indicating a reluctance of overweight or obese mothers to understand or admit that their children are overweight ${ }^{(8)}$. Alternatively, overweight mothers may also believe that young children may grow out of being overweight or that increased fatness signifies health ${ }^{(35)}$.

Another factor that was found to be significantly associated with an increased likelihood of maternal verbal underestimation of children's weight status was when the child's primary caregiver was a person other than the mother or grandparents (i.e. an aunt, older siblings, etc). None of the previous relevant studies have ever examined this variable as a potential correlate of parental perceptions. As many working mothers these days usually leave another person in charge of their children's upbringing (i.e. a nanny, grandparents, an aunt, older siblings, etc.), the limited time that these mothers spend with their children may restrict their ability to identify their children's actual weight or height correctly. Finally, the results of the present study support the idea that parents are more likely to visually underestimate the weight status of boys than of girls ${ }^{(14,16,36)}$. Although this may relate to sex differences in body composition, it seems more likely to reflect social values as well. Mothers may be more sensitive to weight and body image issues in girls, whereas bigger boys may be seen as having a physical advantage.

The present study has certain potential limitations. First, only $12 \cdot 6 \%$ of mothers were found to be obese. This could be attributed to deliberate under-reporting of weight, which is a common weakness when using selfreported data. Furthermore, the use of photographs that were based on the 1979 NCHS growth charts ${ }^{(31)}$ to visually classify children's weight status could represent another possible limitation of the present study, since childhood obesity has more than doubled during the past three decades ${ }^{(37)}$. Although there was no other valid visual instrument that could be used alternatively in the present study, this limitation could provide one possible explanation for the higher maternal underestimation rates derived from the use of the visual compared with the verbal classification instrument. This could also be attributed to the fact that the photographs used in the present study represented pre-pubescent children, whereas the sample also included children at a higher stage of sexual maturation. Nevertheless, no significant association was found between the Tanner stage and the accuracy of maternal perceptions of children's weight status with the use of photographs.

To conclude, the verbal instrument used in the present study to assess maternal perceptions of their children's actual weight status was found to be more accurate compared with the visual one. Considerably higher errors of underestimation occurred for overweight and obese children, whereas overweight mothers and those with a lower educational level were more likely to underestimate their children's weight status. Furthermore, children's male gender and a nanny or a person other than the mother as the child's primary caregiver were found to increase the odds of both visual and verbal maternal underestimation of children's weight status, respectively. These findings have important public health implications since - given that maternal perception might be an indirect determinant of their children's body weight - any interventional programme to induce weight loss is unlikely to be successful without parental involvement. Moreover, even though perceptions are strongly influenced by culture, the fact that we live in an era of globalization makes the findings of the present study relevant to public health experts who deal with childhood obesity in almost all developed countries worldwide. The current findings might be even more relevant to minority groups and populations of lower socioeconomic level living in developed countries, for whom insufficient awareness on health issues makes the risk of body weight underestimation even greater. Thus, the attempt to combat childhood obesity in clinical and public health settings might be more successful if future research also focuses on detecting mothers who are more likely to misperceive their children's weight status. Educating these mothers to classify their children's weight status correctly might be a key factor for the implementation of successful childhood obesity prevention initiatives.

\section{Acknowledgements}

The present research received no specific grant from any funding agency in the public, commercial or not-for-profit sectors. None of the other authors had any personal or financial conflict of interest to declare. G.M. and Y.M. were responsible for the study design, the supervision of the field study and the performance of the statistical analyses. All authors contributed to data collection, data management and database preparation as well as to interpretation of the data, writing and revision of the manuscript and approval of the final version submitted for publication. The authors thank Anastasia Vandorou, Odysseas Androutsos, Evangelia Grammatikaki, Maria Kantilafti, Anthi Naoumi, Aliki E. Farmaki, Aikaterini Siopi, Eleni Z. Papadopoulou, Elpida Voutsadaki, Fanouria Chlouveraki, Konstantina Maragkopoulou, Efstathoula Argyri and Angeliki Giannopoulou for their valuable contribution to the completion of the study. 


\section{References}

1. International Obesity Taskforce (2008) Global trends in Childhood Overweight. http://www.iaso.org/publications/ trackingobesity/global-trends-childhood-overweight/ (accessed May 2011).

2. Freedman DS, Dietz WH, Srinivasan SR et al. (1999) The relation of overweight to cardiovascular risk factors among children and adolescents: the Bogalusa Heart Study. Pediatrics 103, 1175-1182.

3. Field AE, Cook NR \& Gillman MW (2005) Weight status in childhood as a predictor of becoming overweight or hypertensive in early adulthood. Obes Res 13, 163-169.

4. Nanchahal K, Morris JN, Sullivan LM et al. (2005) Coronary heart disease risk in men and the epidemic of overweight and obesity. Int J Obes (Lond) 29, 317-323.

5. Caprio S \& Genel M (2005) Confronting the epidemic of childhood obesity. Pediatrics 115, 494-495.

6. Davison KK \& Birch LL (2001) Childhood overweight: a contextual model and recommendations for future research. Obes Rev 2, 159-171.

7. de Onis M (2004) The use of anthropometry in the prevention of childhood overweight and obesity. Int $J$ Obes Relat Metab Disord 28, Suppl. 3, S81-S85.

8. Baughcum AE, Chamberlin LA, Deeks CM et al. (2000) Maternal perceptions of overweight preschool children. Pediatrics 106, 1380-1386.

9. Carnell S, Edwards C, Croker H et al. (2005) Parental perceptions of overweight in 3-5 y olds. Int J Obes (Lond) 29, 353-355.

10. Rhee KE, De Lago CW, Arscott-Mills T et al. (2005) Factors associated with parental readiness to make changes for overweight children. Pediatrics 116, e94-e101.

11. Genovesi S, Giussani M, Faini A et al. (2005) Maternal perception of excess weight in children: a survey conducted by paediatricians in the province of Milan. Acta Paediatr 94, 747-752.

12. Harnack L, Lytle L, Himes JH et al. (2009) Low awareness of overweight status among parents of preschool-aged children, Minnesota, 2004-2005. Prev Chronic Dis 6, A47.

13. Jansen W \& Brug J (2006) Parents often do not recognize overweight in their child, regardless of their sociodemographic background. EurJ Public Health 16, 645-647.

14. Jeffery AN, Voss LD, Metcalf BS et al. (2005) Parents' awareness of overweight in themselves and their children: cross sectional study within a cohort (EarlyBird 21). BMJ 330, 23-24.

15. Cattaneo A, Monasta L, Stamatakis E et al. (2009) Overweight and obesity in infants and pre-school children in the European Union: a review of existing data. Obes Rev 11, 389-398.

16. Maynard LM, Galuska DA, Blanck HM et al. (2003) Maternal perceptions of weight status of children. Pediatrics 111, 1226-1231.

17. Muhammad NA, Omar K, Shah SA et al. (2008) Parental perception of their children's weight status, and its association with their nutrition and obesity knowledge. Asia Pac J Clin Nutr 17, 597-602.

18. Tschamler JM, Conn KM, Cook SR et al. (2010) Underestimation of children's weight status: views of parents in an urban community. Clin Pediatr (Phila) 49, 470-476.
19. Watkins MG, Clark KM, Foster CM et al. (2007) Relationships among body mass index, parental perceptions, birthweight and parental weight after referral to a weight clinic. J Natl Med Assoc 99, 908-913.

20. Killion L, Hughes SO, Wendt JC et al. (2006) Minority mothers' perceptions of children's body size. Int J Pediatr Obes 1, 96-102.

21. Warschburger P \& Kroller K (2009) Maternal perception of weight status and health risks associated with obesity in children. Pediatrics 124, e60-e68.

22. Oude Luttikhuis HG, Stolk RP \& Sauer PJ (2010) How do parents of 4- to 5-year-old children perceive the weight of their children? Acta Paediatr 99, 263-267.

23. Chaimovitz R, Issenman R, Moffat $\mathrm{T}$ et al. (2008) Body perception: do parents, their children, and their children's physicians perceive body image differently? J Pediatr Gastroenterol Nutr 47, 76-80.

24. Eckstein KC, Mikhail LM, Ariza AJ et al. (2006) Parents' perceptions of their child's weight and health. Pediatrics 117, 681-690.

25. He M \& Evans A (2007) Are parents aware that their children are overweight or obese? Do they care? Can Fam Physician 53, 1493-1499.

26. Jensen DM, Damm P, Sorensen B et al. (2003) Pregnancy outcome and prepregnancy body mass index in 2459 glucose-tolerant Danish women. Am J Obstet Gynecol 189, 239-244.

27. Huang JS, Becerra K, Oda T et al. (2007) Parental ability to discriminate the weight status of children: results of a survey. Pediatrics 120, e112-e119.

28. Cole TJ, Bellizzi MC, Flegal KM et al. (2000) Establishing a standard definition for child overweight and obesity worldwide: international survey. BMJ 320, 1240-1243.

29. Cole TJ, Flegal KM, Nicholls D et al. (2007) Body mass index cut offs to define thinness in children and adolescents: international survey. BMJ 335, 194.

30. Tanner JM (1955) Growth at Adolescence. Oxford: Blackwell Scientific.

31. Truby H \& Paxton SJ (2002) Development of the Children's Body Image Scale. Br J Clin Psychol 41, 185-203.

32. Hamill PV, Drizd TA, Johnson CL et al. (1979) Physical growth: National Center for Health Statistics percentiles. Am J Clin Nutr 32, 607-629.

33. Akerman A, Williams ME \& Meunier J (2007) Perception versus reality: an exploration of children's measured body mass in relation to caregivers' estimates. J Health Psychol 12, 871-882.

34. Suzanne Goodell L, Pierce MB, Bravo CM et al. (2008) Parental perceptions of overweight during early childhood. Qual Health Res 18, 1548-1555.

35. Baughcum AE, Burklow KA, Deeks CM et al. (1998) Maternal feeding practices and childhood obesity: a focus group study of low-income mothers. Arch Pediatr Adolesc Med 152, 1010-1014.

36. Campbell KJ, Crawford DA \& Ball K (2006) Family food environment and dietary behaviors likely to promote fatness in 5-6 year-old children. Int J Obes (Lond) 30, $1272-1280$.

37. Lobstein T, Baur L \& Uauy R (2004) Obesity in children and young people: a crisis in public health. Obes Rev $\mathbf{5}$, Suppl. 1, S4-S85. 